\title{
Primary pulmonary artery sarcoma: intra-operative similarity to pulmonary embolus
}

\author{
G.A. Berg ${ }^{*}$, B.N.A. Hamid ${ }^{2}$, W.E. Kenyon ${ }^{2}$ and M.J. Drakeley ${ }^{1}$ \\ ${ }^{\prime}$ Department of Cardiothoracic Surgery and ${ }^{2}$ Department of Pathology, Regional Cardiothoracic Centre, \\ Broadgreen Hospital, Thomas Drive, Liverpool, UK.
}

\begin{abstract}
Summary: An infarcted right lower lobe, thought to be due to a thrombotic pulmonary embolus, was resected in a 48 year old man. Subsequent examination of the lobe unexpectedly showed the infarction to be secondary to a primary sarcoma of the pulmonary artery.
\end{abstract}

\section{Introduction}

Primary pulmonary artery sarcoma is a rare tumour which most commonly presents with progressive right heart failure, closely resembling a clinical picture of chronic thromboembolism. ${ }^{1,2}$ Acute pulmonary embolism can also be simulated as cough, chest pain and haemoptysis are frequent symptoms. ${ }^{3}$ This communication reports a sarcoma of a lobar pulmonary artery, which had the intra-operative appearance of a thromboembolism, and discusses the difficulty in diagnosing and treating this tumour.

\section{Case report}

A 48 year old man underwent initial investigation of a possible primary bronchial carcinoma in January, 1983. He had a six week history of pleuritic right sided chest pain associated with dyspnoea of effort but was otherwise asymptomatic. He had smoked 20 cigarettes a day for 25 years.

He was well nourished with no signs of heart failure and there were no murmurs. A chest X-ray showed a round mass in the right lower lobe, a small pleural effusion and some prominence of the lower pole of the right hilum. Tomograms confirmed that there was a solid mass in the lower lobe. Pleural aspiration revealed blood-stained fluid with no neoplastic cells. Liver function tests, biochemical and haematological indices were all normal as were his electrocardiogram and pulmonary function tests. Bronchoscopic examination was also normal.

At an exploratory right thoracotomy, the parietal

\footnotetext{
*Correspondence and present address: G.A. Berg, F.R.C.S., Ed., Department of Cardiothoracic Surgery, Royal Infirmary, Alexandra Parade, Glasgow, G31 2ER, UK.

Accepted: 19 November 1986
}

pleura was found to be over $1 \mathrm{~cm}$ thick in places. A layer of fibrin had to be decorticated before the underlying lower lobe could be palpated. A boggy mass and a separate triangular infarction were present in the lower lobe. Tissue from the mass was sent for frozen section and whilst awaiting the result, the pulmonary artery to the lower lobe was dissected free and was found to be solid distally. The right main pulmonary artery felt normal.

Frozen section revealed that the mass was infarcted lung, subsequently confirmed on paraffin section. A working diagnosis of pulmonary embolus was made and so the pulmonary artery to the lower lobe was clamped and opened in an attempt to perform an embolectomy. The artery was found to be occluded with a mass which had the appearances of an organizing thrombus. This was removed from the proximal part of the artery although it was thought that a small amount was left behind attached to the intima. It was not possible to remove the mass from the distal branches of the lower lobe artery, therefore a right lower lobectomy was performed. The patient made an unremarkable post-operative recovery.

Pathological examination unexpectedly showed a greyish glistening tumour occupying the lumen of the lobe's main pulmonary artery and extending distally into the peripheral arteries (Figure 1). No extension into lung parenchyma was identified. The visceral pleura was covered by fibrinous exudate and there were two underlying wedge shaped infarctions.

Histological examination showed that the lesion was composed of interlacing bundles of spindle cells with marked pleomorphism and hyperchromasia. There were frequent and abnormal mitoses as well as tumour giant cells (Figure 2). The appearances were those of a sarcoma with no definite indication of its histiogenesis. The intimate association of the lesion 


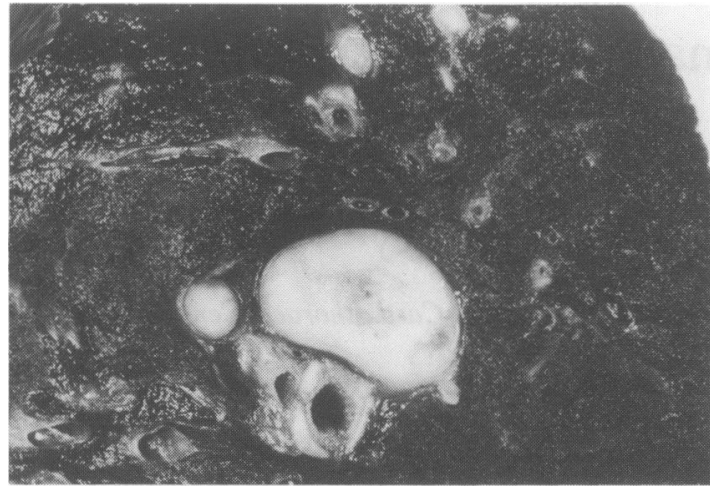

Figure 1 Tumour occupying branches of the pulmonary artery to the lower lobe.

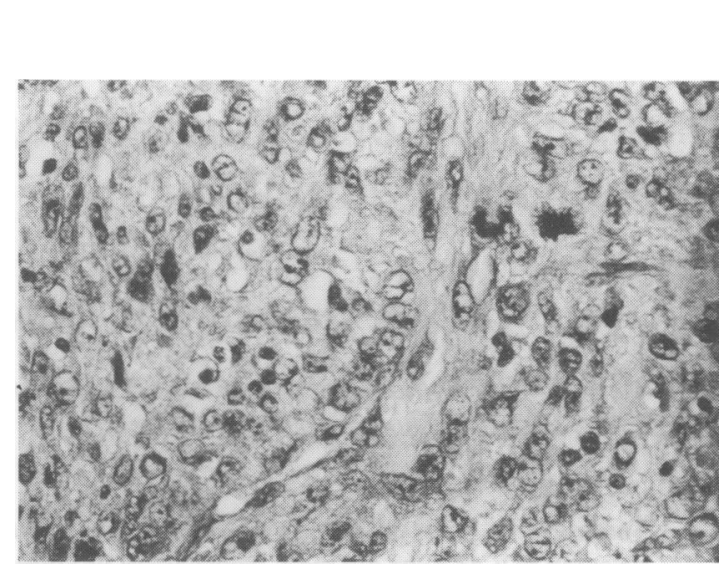

Figure 2 Histological section showing spindle cells, Giant cells and frequent mitoses. (H \& E $\times 400)$. with the vessel wall and the absence of extravascular extension were in keeping with a primary pulmonary artery sarcoma.

The patient declined a completion pneumonectomy but was treated by irradiation to his mediastinum. He remained well for 12 months until he presented with an expanding mass in the posterior border of his right external oblique muscle. This was excised and was shown to be a secondary deposit, histologically similar to the primary. He received irradiation to this site and again remained well until July 1985 when he developed a local recurrence in the scar. Despite therapy in the form of cyclophosphamide and prednisolone, the secondary deposit increased in size. The patient died 40 months after surgery during which time he had shown no radiological evidence of an intra-thoracic recurrence. Consent for a post-mortem examination was not obtained.

\section{Discussion}

Primary malignant tumours arising from the pulmonary trunk or main pulmonary artery are extremely rare. The tumour was first described in 1923 by Madelstamm ${ }^{4}$ and a comprehensive review of a further 60 cases, reported in the literature, was made more recently by Bleisch \& Kraus. ${ }^{5}$ All of their reported cases showed origin of tumour in the pulmonary trunk with direct extension along the flow of blood to the left or right main pulmonary artery. The authors postulate that these tumours arise from tissues derived from the bulbus cordis. In this reported case, although the tumour could have arisen from the right main pulmon- ary artery, repeat pulmonary angiography showed that the trunk was clear of tumour.

Microscopically, over $90 \%$ of tumours have been 을 described as sarcomas containing spindle cells. Leiomyosarcoma, rhabdomyosarcoma, fibrosarcoma and myxosarcoma have also been described.

The prognosis is poor and the diagnosis is usually $\vec{\bullet}$ made at post-mortem examination ${ }^{6}$ but sever@ patients have had attempted removal by pneumonec tomy. ${ }^{3,6,7}$ Sixty seven per cent of patients in Bleisch series had metastatic spread along the pulmonary arteries to the lung periphery, producing a clinical picture which may be mistaken for right heart failure due to multiple thrombotic pulmonary emboli. Extrathoracic metastases are uncommon and have never before been described in muscle. Most patients who underwent attempted resection of their tumours died within 12 months even with adjuvant chemotherapy or radiotherapy. ${ }^{5}$ Diagnosis is usually made by pulmonary angiography which shows a filling defect but one tumour has been diagnosed with two-dimensional echocardiography. ${ }^{8}$

The poor prognosis appears to be related to the site of the tumour, its early intra-thoracic embolic spread and the delay or inability to make the correct diagnosis. Previous reports have emphasized that in the differential diagnosis of right heart failure, associated with the radiographic appearance of pulmonary o emboli, pulmonary artery neoplasms with multiple N lung secondaries should be considered. ${ }^{9}$ This report demonstrates that intra-operatively, it may also be technically difficult to differentiate this tumour from a thrombotic pulmonary embolus. 


\section{References}

1. Friedman, H.M. \& Smith, C.K. Leiomyosarcoma of the pulmonary artery. JAMA 1968, 203: 809.

2. Thijs, L.G., Kroon, T. \& Leeuwen, T. Leiomyosarcoma of the pulmonary trunk associated with pericardial effusion. Thorax 1974, 29: 490-494.

3. Sethi, G.K., Slaven, J.E., Kepes, J.J. et al. Primary sarcoma of the pulmonary artery. J Thorac Cardiovasc Surg 1972, 63: 587-593.

4. Madelstamm, M. Ueber primaere Neubildungen des Herzens. Virchows Arch (A) 1923, 245: 43-54.

5. Bleisch, V. \& Kraus, F. Polypoid sarcoma of the pulmonary trunk: analysis of the literature and report of a case with leptomeric organelles and ultra structural features of a rhabdomyosarcoma. Cancer 1980, 46: 314324.
6. Shmookler, B.M., Marsh, H.B. \& Roberts, W.C. Primary sarcoma of the pulmonary trunk and/or right or left pulmonary artery - a rare cause of obstruction to right ventricular outflow; report on two patients and analysis of 35 previously described patients. Am J Med 1977, 63: 263-272.

7. Jacques, J. \& Barclay, R. The solid sarcomatous pulmonary artery. Br J Dis Chest 1964, 54: 217-220.

8. Wright, E., Wellons, H. \& Martin, R. Primary pulmonary artery sarcoma diagnosed non-invasively by twodimensional echocardiography. Circulation 1983, 67: 459-462.

9. Olsson, H.E., Spitzer, R.M. \& Erston, W.F. Primary and secondary pulmonary artery neoplasia mimicking acute pulmonary embolism. Radiology 1976, 118: 49-54. 